Ebisu Ebisu

Études japonaises Études japonaises

47 | printemps-été 2012

Catastrophes du 11 mars 2011, désastre de

Fukushima : fractures et émergences

\title{
Les démons du Tōhoku
}

\section{Christine Lévy}

\section{(2) OpenEdition}

10 Journals

Édition électronique

URL : http://journals.openedition.org/ebisu/199

DOI : 10.4000/ebisu.199

ISSN : 2189-1893

Éditeur

Institut français de recherche sur le Japon à la Maison franco-japonaise (UMIFRE 19 MEAE-CNRS)

Édition imprimée

Date de publication : 1 juin 2012

Pagination : 7-9

ISSN : 1340-3656

\section{Référence électronique}

Christine Lévy, «Les démons du Tōhoku », Ebisu [En ligne], 47 | printemps-été 2012, mis en ligne le 13 mars 2014, consulté le 22 septembre 2020. URL : http://journals.openedition.org/ebisu/199 ; DOI : https://doi.org/10.4000/ebisu.199 


\section{Introduction}

\section{Les démons du Tōhoku}

Ce numéro d'Ebisu est né d'un appel à contributions lancé en juin 2011. Il entendait rendre compte, entre autres, de tout ce qui pouvait montrer la montée d'un refus de l'ordre des choses. Trois mois après, le 19 septembre 2011, soixante mille personnes se rassemblaient à Tokyo au parc Meiji pour dire leur refus du nucléaire. Parmi les orateurs, Mutō Ruiko 武藤類子, une militante antinucléaire de longue date à Fukushima, y prononçait un discours émouvant, aussitôt traduit en chinois, en anglais et en français pour être diffusé sur les sites internet ${ }^{1}$. Tout était dit dans ce discours, l'essentiel en termes simples et clairs ; aux côtés de nombreux militants antinucléaires, beaucoup de personnes manifestaient pour la première fois de leur vie, pour exprimer à la fois leur solidarité envers les personnes sinistrées, leur colère contre un système qui s'est montré défaillant, leur rejet de l'irresponsabilité et leur refus de léguer aux générations futures le fardeau du nucléaire. La peine d'une perte irrémédiable, celle de la nature, du pays natal et de ses trésors, se doublait d'une tristesse de voir les liens humains se briser et se déchirer, pris en tenaille entre la " campagne de sécurité " aussitôt déployée par les autorités de l'État et la direction de Tepco d'une part, et d'autre part l'angoisse qui tourmentait chacun en son for intérieur dans les décisions immédiates à prendre. Parmi ces dilemmes, se taire ou protester ; une certitude, que l'accident n'était pas terminé, qu'il était loin d'être maitrisé, que

1. Voir : <http://www.youtube.com/watch?v=IH-66MqFTdw> (consulté le 8 mai 2012). 
trop de vérités avaient été cachées, et le sentiment d'être mis au rebut ont eu raison de l'accablement et de la tristesse. «Ne nous prenez pas pour des idiots ", "Ne nous volez pas nos vies " ont sonné comme le cri du cœur de ces habitants du Tōhoku, et du désespoir des paysans de voir leur terre polluée, des cris de protestations se sont fait entendre ; refusant les nouvelles discriminations et divisions, des voix ont commencé à demander des comptes à l'État et à Tepco. "Nous sommes devenus les démons du Tōhoku, brûlant calmement de fureur ", déclarait Mutō Ruiko.

À cette terrible blessure du tsunami qui a emporté près de vingt mille vies s'est ajouté ce drame écologique et politique, source de suspicion, de meurtrissure qui empêchaient chacun de sortir de la culpabilité lancinante.

Depuis, la centrale de Tomari à Hokkaidō fut mise à l'arrêt le 6 mai 2012, et désormais aucun des réacteurs nucléaires implantés sur le sol japonais, au nombre de 54, n'est en activité, bien que la question du redémarrage ou non de la centrale d'Ōi dans le département de Fukui reste en suspens. Des voix de plus en plus nombreuses prônent la sortie du nucléaire, même si d'autres forces - nationales et internationales - travaillent à son maintien. Le mythe de la sécurité du nucléaire s'est effondré, le primat de l'économie ne peut plus légitimer le sacrifice d'une partie de la population, et l'espoir de voir de leur vivant la sortie totale du nucléaire gagne de nouvelles personnalités ou responsables politiques. La question de l'adoption de lois protectrices contre l'irradiation est enfin en cours de discussion au sein de l'assemblé législative. Et les revendications des femmes de Fukushima pour la reconnaissance du droit à l'évacuation de toutes les familles ne restent pas complètement inaudibles. Les enjeux politiques de l'avenir sont là. Or nous ne pouvons que constater une lenteur manifeste dans la prise des décisions politiques, juridiques et humanitaires, pourtant si urgentes. Nous ne pouvons oublier tous les problèmes liés à la reconstruction, autant matérielle que morale, de toute une région dévastée par le malheur.

Chacun des articles présentés ici interroge en filigrane le lien de la catastrophe naturelle à la catastrophe humaine, ce qui fait écho à la culture et à l'histoire du pays, la nature des liens tissés à travers le passé et le présent, et ce que ceux-ci permettent d'envisager à l'avenir.

Nous remercions ici tous les contributeurs/trices grâce à qui nous pouvons faire état de la situation et de la multiplicité des enjeux, et offrir des outils d'analyse, rendre compte des débats en cours depuis le 11 mars. La 
traduction que nous proposons du texte de Kamata Satoshi 鎌田慧, «Du refus à la solidarité », paru en octobre 2011 dans la revue Gendai shisō 現代思想, apportera au lecteur un témoignage direct et à travers l'histoire d'un engagement personnel, un éclairage saisissant tant sur l'historique du mouvement antinucléaire au Japon que sur ses enjeux actuels. Enfin, avec le texte de Thierry Ribault, auteur de l'appel à contributions et co-responsable de ce numéro, nous présentons ses pistes de réflexions et ses perspectives théoriques, sur la question de la protection notamment, qui iront nourrir les débats et les recherches indispensables à l'avenir.

Ce numéro est aussi notre manière à nous tous de rendre hommage à tous ceux qui luttent pour sortir de leur détresse, à ceux qui leur sont venus en aide, et d'exprimer un sentiment de solidarité au-delà des clivages que cette catastrophe a fait naître.

Le 8 mai 2012,

Christine LÉVY

Nous tenons à remercier tous les membres du comité de rédaction, en particulier Jennifer Hasae pour le travail éditorial, ainsi que Marc Humbert, ancien directeur de la publication, qui a pris l'initiative de lancer ce numéro. 